\title{
Interval Exercise Training Affects Expression of MCT-1, GLUT-1, PFK-1 and p53 as Key Metabolic Regulators on Azoxymethane-Induced Experimental Colon Cancer
}

Hossein Shirvani ( $\nabla$ shirvani7911@gmail.com )

Baqiyatallah University of Medical Sciences https://orcid.org/0000-0002-9465-0199

Behzad Bazgir

Baqiyatallah University of Medical Sciences

Mostafa Rahimi

Shahrekord University

Amin Isanejad

Shahed University

Mohammad Samadi

Baqiyatallah University of Medical Sciences

Vahid Sobhani

Baqiyatallah University of Medical Sciences

Ali Mohammad Alizadeh

Tehran University: University of Tehran

Ehsan Arabzadeh

Baqiyatallah University of Medical Sciences

Research

Keywords: Exercise, Glucose, Lactate, Tumor

Posted Date: February 2nd, 2021

DOI: https://doi.org/10.21203/rs.3.rs-151527/v1

License: (c) (i) This work is licensed under a Creative Commons Attribution 4.0 International License. Read Full License 


\section{Abstract \\ Background}

Physical activity is one of the modulators of the cancer risk and survival factors. Therefore, the aim of this study was considering of pre and post interval exercise training on expression of MCT-1, GLUT-1, PFK1 and p53 as a key metabolic regulators in azoxymethane-induced experimental colon cancer

\section{Methods}

Forty-eight male BALB/c mice were equally randomized into 6 groups: I: control (C); healthy animals, II: Exercise (E), III: tumor induction ( $T$ ); animals received AOM for inducing colon cancer, IV: $A O M+$ exercise (TE); animals with colon cancer underwent 8 weeks of the interval training protocol after tumor establishment, V: exercise + AOM (ET); animals received exercise protocol one week before AOM consumption, and $(\mathrm{VI})$ exercise + $\mathrm{AOM}+$ exercise (ETE); animals received exercise protocol one week before and after AOM consumption (about 15 weeks) Groups III-VI were weekly-received AOM (as a carcinogenic agent, $10 \mathrm{mg} / \mathrm{kg}$ s.c) in three consecutive weeks to induce colon cancer. Interval exercise training was begun at 16-18 m/min, 10-14 min, 5 days/week for 6 weeks.

\section{Results}

The results showed that the tumor significantly increased mRNA and protein of MCT1 in the tumor group compared to the control group $(p<0.001)$, Also, exercise before and after tumor induction reduced MCT1 (and other glucose regulators) in the colon (respectively: $p<0.02$ and $p<0.01$ ). While the p53 gene decreased significantly in the tumor group compared to the control group $(p<0.01)$. Exercise before tumor induction and after tumor induction increased significantly ( $p<0.01$ and $p<0.04$ respectively) in p53 compared to the tumor group. ETE group also downregulate the expression of glucose metabolism genes in colon tumor $(p<0.05)$.

\section{Conclusion}

Long-term aerobic interval exercise (pre, post, pre\&post tumor) can contribute to inhibition of tumor progression and treatment against colon cancer. It seems that these preventive and treatment effects exercise training can attribute to the regulation of lactate and glucose transporters by up-regulation of p53 colorectal cancer cells.

\section{Key Points}

Exercise before and after tumor induction significantly increased p53 in colon. 
Exercise pre and post tumor induction down regulate the expression of glucose metabolism genes in colon tumor

\section{Introduction}

In today's world, with increases risk factors for health, the incidence of various cancers has increased dramatically [1]. One of the common cancers in men is colon cancer [2]. Each year, 1 million people in the world diagnosed with colon cancer [3]. Alteration of cellular energy metabolism is one of the most important signs of cancer cells [4]. Mitochondrial dysfunction, loss of tumor suppressors, hypoxia microenvironments, and oncogene-driven metabolic reprogramming considered as early abnormal events in energy metabolism of cancer cells [5]. In more than $70 \%$ of human cancers, glycolytic genes are often overexpressed [6], Because cancer cells mainly produce energy by increasing glycolysis (the amount of glycolysis in the cancer cell is 200 times higher than normal cells), that it results production of lactate in the cytosol of the cell, even in the presence of oxygen. These conditions provide bioenergetics and biosynthetic needs to support rapid proliferation in cancer cells. This operation called Warburg's effect in oncology conditions [7].

According to the Warburg hypothesis, cancer cells, instead of oxidative phosphorylation, select aerobic glycolysis as the main method of glucose metabolism. New studies on cancer therapy have been targeted inhibitor of aerobic glycolysis. Also other new drugs, target enzymes, and metabolic enhancers that have great potential to block the progression of cancer [8]. The consumption of glucose is a limiting step for the aerobic glycolysis of cancer cells. In cancer cells, glucose are absorbed from the microenvironment into the cytoplasm from plasma membrane occurs with facilitated transfer of glucose by transporter / solute carrier (GLUT / SLC2A). Under hypoxic conditions, cancer cells often express the high amount of metabolic regulators such as GLUTs including GLUT1 and GLUT3. About the GLUT1, numerous studies have examined the inhibitory role of this marker in treating of cancer[9]. The GLUT-1 has a potential target for cancer therapy, as inhibiting the expression of this protein is associated with a decrease in tumor growth[10]. Various studies have also shown that flavonoids can have an inhibitory role in GLUT-1 and inhibit tumor growth[11]. Consequently, inhibiting this protein in cancer cells can be effective in inhibiting of tumor growth. After transferring of glucose into the tumor cell, the glycolysis pathway triggered, and just in three phases of this pathway be control by key enzymes, one of the most important of these enzymes being PFK. Two types of PFK include PFK-1 and PFK-2. It stated that under the conditions of colon, berest, ovarian and thyroid cancer, the pattern of expression of PFK increases[12]. However, studies limited on the effect of various factors on inhibiting and reducing the activity of this enzyme under conditions of tumor colon.

At the end of the aerobic glycolysis pathway, pyruvate is converted to lactic acid by lactate dehydrogenase-A (LDHA). The accumulation of lactic acid in cancer cells promotes the transfer of lactic acid by the Monocarboxylate transporter (MCT), especially MCT1[13] and MCT4[14]. Disruption of MCT as a metabolic regulator has been observed in the pathologic and tumor conditions. In the tumor, MCTs control the transfer of lactate and other carboxylates between glycolytic and oxidative in cancer cells. It 
reported that up regulation of MCT1 and MCT4 occur in several solid tumors such as glioblastoma, chest, colon, ovarian, and liver cancer[15]. Inhibition of MCT1 reduces lactate access for cellular oxidative activity and forcing them to use glucose. This leads to glucose starvation and cell death of hypoxia cells and subsequently leads to stopping tumor growth[16]. MCT1 and MCT4 have potential targets for cancer therapy. Boidot et al. (2011) revealed a direct relationship between the function of p53 and the expression of MCT1 in cancer therapy[17].

P53 is one of the most important inhibitors of cancer, and it has been suggested that the reduction of this gene is effective in the further development of cancers[18]. The p53 tumor-inhibiting marker prevents the development of cancer by various mechanisms, including induction of apoptosis, stopping the cell cycle. Recently, the relationship between p53 and tumor metabolism has been shown[19]. Under hypoxia conditions, p53 deficiency, promotes the expression of MCT1 and transmission of lactate produced from rising glycolytic flocculation. p53 interacts directly with the promoter of the MCT1 gene and it changed MCT1 mRNA stabilization. And it can be effective in treating of cancer. It has been arguing that p53 deficiency promotes the expression of MCT1 in hypoxia conditions, thereby facilitating the release of lactate, which leads to an increase in glycolytic flux in cancer cell. When p53 is active, expression of MCT1 is very low[17]. In other words, p53 controls the levels of lactate in tumor cells through the repression of MCT1[17]. Studies have shown that physical activity with effect on these enzymes and metabolic controllers able to prevent and control the growth of the tumor[20] (Fig. 1).

It has been argued that exercise reduce the risk of breast, colon, and prostate cancers [20-22]. In other words, exercise can prevent and protect from cancers. Regarding the preventive role of exercise training, it is stated that Exercise before inducing injuries such as stroke and tumors can minimize the subsequent damage caused by these injuries, which is known as exercise preconditioning. In relation to tumor cancer it is stated that expression of Netrin-1 and its receptors deleted in colon cancer (DCC) and uncoordinated gene 5B (Unc5B), known mediators of neural and vascular activities, are also regulated in the exercise preconditioning in vascular activity[23]. In addition, Epidemiological evidence supports a protective effect of physical activity after colon cancer. For example, regular moderate to vigorous physical activity was shown to be associated with a lower risk for developing proximal (24\%) and distal (23\%) colon in colon cancer[24]. In addition to numerous studies that showed that exercise with an effect on myokine and catecholamine were effective in preventing of cancer, it also directly regulates tumor growth[25]. Due to the very high glycolytic activity of cancer cells, known as one of the main symptoms of cancer cells, it may be one of the treatment options in cancer therapy[26]. Evidence indicates that exercise may also be directly linked to the control of tumor biology by targeting of tumor glycolysis[27]. Physical activity is effective in increasing metabolism of total body and intracellular metabolism. Several studies have suggested that tumors, including colon tumors, which inherently have a high metabolism rate, can be affected of under the influence of energy stress caused by exercise such as fasting and caloric restriction [28-30]. In recent studies, Devin et al (2019) show that acute high intensity interval exercise (HIIE) reduced colon cancer cell number in vitro and promoted increases in inflammatory cytokines immediately following exercise.[20]. Hojman et al. (2018) suggested that regular exercise could reduce cancer by controlling tumor growth. Consequently, the control of caloric intake of the tumor through exercise may 
be a more feasible approach for lowering and control of cancer[27]. Because one of the major effects of exercising is targeting the specific metabolism of tumor cells(namely the Warburg-type highly glycolytic metabolism) [31] (Fig. 1). Also tumors metabolism as well as the tumor host interaction may be selectively influenced by exercise training, dependent on exercise intensity, duration, frequency, and mode [32].

Studies with multiple exercise interventions including voluntary wheel running, running on treadmill, and swimming in rodents showed that exercise training is effective in reducing tumor incidence, tumor growth, and metastasis in animal models By inducing cancer Or genetically [33] Some studies also suggested that some cancers with a genetic background have a different sensitivity to exercise[34]. For example, genetic defect in p53 is an animal model with breast cancer did not show a regulation with physical activity[35]. In 2008, McTiernan (2008) suggested that physical activity can be effective in protecting cancer by reducing the risk factor associated with cancer, such as reducing inflammatory markers[33]. In relation to the effect of exercise before and after tumor, Different research has been done on human and animal samples. Aveseh et al. (2015) showed that exercise can affect in reducing lactate levels in the tumor through impact on LDH [36]. However, About the MCT1 changes in colon cancer with exercise studied is limited. Therefore, the aim of this study was to investigating the preventive (preconditioning) and treatment effects of exercise training with considering key marker involved in colon cancer cells metabolism.

\section{Results}

\section{Body weight, colon and tumor changes}

The results of weight changes showed that induction of a colon tumor caused a significant increase in animal weight $(p<0.05)$. Although pre- and post-exercise with tumor and ETE caused weight loss compared to the tumor group, these changes were not significant $(p>0.05)$, (table 2$)$.

Examination of the colon's weight changes also revealed that the tumor group had a significant increase in colon weight $(p<0.05)$, while only the ETE group showed a significant decrease compared to the tumor group $(p<0.05)$, (table 2$)$.

The results of colon length changes also showed that, the tumor group had a significant reduction in colon length compared to the colon group. But compared to the colon group, the ET and ETE groups had a longer colon length $(p<0.05)$, (table 2$)$.

Examination of the tumor size also showed that TE and ETE groups showed a significant decrease in tumor volume compared to tumor group $(p<0.05)$, (table 2$)$.

\section{mRNA and protein of MCT-1 in colon}

The results of the MCT-1 gene expression showed that tumor induction caused a significant increase in MCT-1 mRNA compare to healthy control group $(p<0.05)$. However, all training groups show a significant 
decrease in MCT-1 mRNA, compare to T group $(p<0.05)$ (Table 3).

The MCT-1 protein content of colon tissue was significantly increased in group $T(P<0.001)$ and TE $(P<$ $0.03)$ groups compared to $C$ group (Fig. 2). However, compared to the T group, the TE $(P<0.01), E T(P<$ $0.02)$ and ETE $(p<0.01)$ groups show a significant decrease of MCT-1 protein content in colon tissue (Fig. 2).

\section{mRNA and protein of GLUT-1 in colon}

The results of GLUT-1 mRNA in colon are also shown in Table 3. These results showed that the tumor group showed a significant increase in GLUT-1 mRNA compared to healthy groups (control and exercise) $(p<0.05)$. However, only TE and ETE groups showed a significant decrease in GLUT-1 mRNA compared to the tumor group (Table 3).

As show in figure 3, The level of GLUT-1 protein significantly increased in T $(P<0.001)$, TE $(P<0.05)$, ET $(P<0.01)$, and ETE $(P<0.01)$ groups compared with healthy control group. It was also found that the TE $(P<0.04)$, ET $(p<0.05)$ and ETE $(P<0.05)$ groups show a significant decrease in colon GLUT-1 compare to T group (Fig. 3).

\section{mRNA and protein of PFK-1 in colon}

similar to the MCT-1 and GLUT-1 gene expression in colon mouse, Also PFK-1 mRNA significantly increased in tumor group compare to control group $(p<0.001)$. However, all training groups showed a significant decrease $(p<0.05)$ in PFK-1 mRNA compared to the tumor group (Table 3).

The protein content of the PFK-1 enzyme in the colon tissue show at figure 4. Based on this results, PFK-1 significantly increased in $T(P<0.001)$ and TE $(P<0.01)$ groups, compared with control group. The $E$, ET $(P<0.01$ for both $)$ and ETE groups $(P<0.02)$ had a significant decrease compared to the $T$ group. Also, the $T$ and the TE groups showed a significant increase compared to the ET and ETE groups $(p<0.05)$.

\section{P53 mRNA}

The results showed that P53 mRNA in colon tissue was significantly decreased in T group compare to healthy control group $(P<0.001)$. As show in figure 5, E $(P<0.001)$, TE $(P<0.05)$, ET group $(P<0.001)$, and ETE $(P<0.05)$ groups significantly increased p53 mRNA in colon tissue compare to $T$ group. Also Exercise group (in healthy mouse) show a significant increase in colon p53 compare to all groups $(p<0.001)$.

\section{Discussion}

Studies on the effects of exercise on cancer have dramatically increased since the 1990s. According to many studies, there is a link between physical activity and a reduction in the risk of colon cancer[37]. It shows that performing of physical activity before the diagnosis of colon cancer reduces about $50 \%$ of the 
risks of this cancer (compared with those who are not active)[38]. In relation to the affecting biological mechanisms on the effect of exercise on colon cancer Studies are limited. Therefore, the purpose of this study was to investigate the effect of aerobic interval training before and after tumor induction in the colon and its effect on the metabolism of lactate and glucose by regulation of $p 53$.

Induction of a colon tumor caused a significant increase in animal weight. Also tumor group had a significant increase in colon weight and colon length. Examination of the tumor size also showed that TE and ETE groups showed a significant decrease in tumor volume compared to tumor group (Table 1). Thus all of this change show induction of tumor compares to control group and prove tumor induction.

Increasing lactate exchange in tumor cells increases the metabolic rate of cancer cells and increases the function of these cells. One of the most important regulators in the transfer of lactate between cancer cells, especially colon cancer, is monocarboxylate transporter, especially MCT-1. According to the results of this study, it was found that the colon tumor has an increase in MCT-1 (Fig. 2). As stated, the increase in MCT-1 in tumor cells leads to an increase in lactate in the tumor microenvironment[39]. Lactate causes expression of HIF-1 in many cancer cells[40] and HIF-1 itself are effective in increasing MCT-1[40]. On the other hand, the increase in lactate for tumor cells is critical because lactate increases cellular migration, angiogenesis, immune escape, and radio resistance[41]. These conditions are suitable for cancer development and reduction of this agent can be a therapeutic target. In the present study, it was determined that aerobic interval training before and after tumor induction significantly decreased MCT-1, that this reduction was higher in the pre-tumor exercise group (ET) (Fig. 2). Stopping or reducing the activity of the MCT-1 Restricted Feeding the cancer cells and places these cells in starvation state. Because in cancer cells, MCTs allow to lactate travel outside the cell and play important role as a mediator of carcinogenesis in other adjacent cancer cells. For this reason, targeting MCTs has a therapeutic role in cancer cells. Inhibition of MCT-1 inhibits tumor growth and carcinogenesis [42, 43]. Therefore, exercise at the cellular level of tumor has limited lactate transport, which is very suitable for cancer treatment. It has been shown that MCT-1 expression regulate by C-MYC in a variety of cancers[42]. However, exercise can reduce the degradation activity of c-MYC[44]. However, in the present study, c-MYC activity was not investigate. Several studies have been conduct on the effect of exercise (in healthy subjects) and colon cancer prevention[27, 45]. In human studies, it has been confirmed that exercises usually accompanied with increasing the quality of life and improve of mental states. These factors although reducing the risk factors of cancer, they effective in improving the physical fitness before the onset of cancer. However, studies are limited in relation to the signaling pathways affecting the MCT-1 with exercise. One of the most effective genes in tumor suppression is p53[46]. Studies show that exercise can cusses increase the tumor suppressor gene, i.e P53[47]. In fact, the mutation of tumor suppressor, directly increases the expression of MCTs, which has been shown this action reprogram the cancer cells towards glycolysis and produce lactate only because the effect of Warburg does not end[47].

P53 controls the levels of lactate in tumor cells by controlling MCT-1[17]. There is a direct correlation between p53 function and MCT-1 expression[39]. Under hypoxic conditions, reduction in p53, promotes the expression of MCT-1 and, export of lactate produced by the rise of glycolytic flux in both vitro and 
vivo. In the present study, changes in p53 mRNA and MCT-1 confirmed this issue. P53 interacted directly with the MCT-1 gene promoter and altered MCT1 mRNA stabilization. Under hypoxic conditions and reducing p53 in tumor cells, NF-KB promotes expression of MCT-1, which increases its levels. Following an increase in glucose consumption in tumor cell, up regulation of MCT-1 under reduced p53 conditions, promotes lactate Import and enhances cell proliferation by increasing mitochondrial respiration[17, 39]. The researchers suggested that increasing the MCT- 1 in conditions of p53 deficiency, allows the tumors to adapt to metabolic needs by facilitating lactate export [17].That these results are consistent with the results of this study. In this study, levels of p53 mRNA were also evaluated. In the healthy groups of the present study, exercise showed a significant increase in colon p53 mRNA compared to healthy animal in control group (Fig. 5). However, tumor induction severely reduced p53 mRNA. Comparing exercise before and after the tumor, was found that exercise before tumor induction had better control in improvement of inhibiting p53 mRNA and increase in p53 mRNA was higher in the ET group. Although exercise after tumor induction (TE) also increased significantly in the p53 mRNA compared to the tumor group, but this increase was lower than the ET group. P53 directly activates the expression of many genes that are used to regulate the cell cycle and apoptosis through binding to p53 DNA-binding sites. P53 plays a vital role in regulating the stopping of the cell cycle and inducing carcinoma cell apoptosis[48]. Thus it can have an anticancer role[49]. P53 inhibits cell cycle progression in the $\mathrm{G} 1$ or $\mathrm{G} 2$ / M phase by inducing $\mathrm{p} 21$ cell cycle inhibitors and enhancing apoptosis by inducing Bax preopoptotic expression[50]. Previous studies have shown that treatment through polyphenols causes p53 up regulation, and p53 can regulate p21, PUMA, and Bax in human colon cancer, which is effective in stopping the tumor[51]. It has been state that p53 activation plays a role in the reduction of glycolysis[52]. Protein P53 inhibits the expression of several carriers of glucose, including GLUT-1 and GLUT4[53].

In consideration of GLUT-1, it was also found that the tumor caused a significant increase in this protein in the colon tissue compared with healthy control and exercise groups (Fig. 3). This increase is normal, due to the high metabolism of cancer cells and the Warburg effect. GLUT-1 is an important isoform in human colon cancer cells[54]. It is stated that under hypoxic conditions, cancer cells often express high amount of GLUT-1. Hypoxic microenvironment of tumor cusses induces of hypoxia induce factor and overexpression of HIF-1a and HIF-1B subunit. HIF-1 activates several signaling pathways in cancerous cells. It has been shown that GLUT-1 levels increase both mRNA levels and protein levels in hypoxia conditions[8]. In other words, HIF-1 causes upregulation expression of GLUT-1 in tumor cell environments[55]. That increased the GLUT-1 tumor group in present study is also justifiable. However, the extent of tumor microenvironment hypoxia, was not investigated in this study. Also, in line with the results of this study, Graziano et al. (2017) showed that GLUT-1 increased in colorectal cancer[56], which increased glucose consumption in cancer cells. These researchers increase of GLUT-1 attributed to increase in RAS oncogenes, because there is a correlation between the positive mutation of the RAS tumor and the expression of the GLUT-1 mRNA[56]. It is stated that inhibition and reduction of GLUT-1 activity in tumor cells through various supplements and medications is effective in the treatment of cancer, and it restricts tumor growth[11]. In the present study, it was found that exercise activity prior to tumor induction (ET) did not significantly decrease in GLUT-1 colon tumor, but when animal's first tumor 
and then exercise (TE), the amount of GLUT-1 tumors decreased, that this decrease was significant (Fig. 2). In experimental examples it is stated that down regulation or inhibition of glycolysis through the GLUT blocker[57]. Which it was confirmed in the present study and exercise can block or decrease the GLUT-1 in tumor colon (in TE group).

Regarding Fig. 3, in addition to the results of GLUT-1 and MCT-1, in the tumor group, a significant increase in PFK-1 was found in the tumor group as a key regulator of glycolysis. However, aerobic interval training before and after tumor induction reduced the amount of this enzyme (significant decrease) (Fig. 4). AMPK activates PFK[58]. Exercise by AMPK can also change the PFK-1 levels in tumor cells. It can also be assumed that changes in levels of tumor PFK-1 with aerobic interval training can be attributed to p53 changes. Because a recent study has shown that p53 causes dermalogue of PFKFB4, one of the enzymes controlling levels of fructose 2-6 bisphosphate, which is the major alloystic regulator of glycolysis[59]. One of the metabolic genes regulated with p53 is TP53-induced Glycolysis and Apoptosis Regulator (TIGAR) that inhibits glycolysis in the allosteric manner through PFK-1 [60]. Inhibition of PFK-1 with TIGAR can indirectly increase the antioxidant capacity by shunting glucose to the pentose phosphate pathway for glutathione biosynthesis. Therefore, TIGAR inhibits tumor DNA by reducing the oxidative damage[60]. On the other hand, a subsequent study has shown that the overexpression of TIGAR in human colon cancers can promote both tumor genesis and the regeneration of normal tissue, demonstrating that cancer cells can also benefit from the metabolic activities of TIGAR[61]. Despite this, the studies are controversial. On the other hand, only p53 mRNA was consider in this study and TIGAR levels of the tumor was not measured. Therefore, it is suggested that the PFK variations in the present study may be influenced by p53 and TIGAR, but it's suggested that in subsequent studies, TIGAR values should also be evaluated.

Aerobic interval training can be countered with progression of Warburg effects of cancer cell. Several evidence has shown that cancer patients who are exercising can had better cope with cancer [62], Because the survival rate of cancer patients increases with physical activity and exercise[63]. However, the molecular and cellular mechanism how that exercise is involved in cancer is still unclear. It has been argued that aerobic exercise improves mitochondrial function and lactate clearance capacity, as it increases fat oxidation capacity and decreases glycolysis[64] in cancer cell. In fact, exercise can have anti-Warburg effects in tumor microenvironment. Christine et al (2002) argued that the biologic effects of exercise on tumor cell can be different and limited research has been done on this issue. The researchers showed that in colon cancer, gastrointestinal transit time is reduced, which exercise can increase the activity of intestinal movements. On the other hand, colon cancer decreases the proportion of prostaglandins. Studies have shown that it high intensity exercise increases the of prostaglandin $F$ activity, that this action suppressing the proliferation of colonic cells, increases the gat mobility, while not increasing PGE2, which can affect the proliferation of colonic cells [65].

\section{Conclusion}


Therefore, aerobic interval training can counteract with metabolic changes and glycolytic metabolism in cancer cells and produce an epigenocytic response that can help to restore p53 mRNA. According to the results of this study, this was confirmed. Because in our study showed that, positive changes of p53 mRNA were consistent with the changes in the metabolic controller of tumor cells, especially the MCT1 with pre-and post-tumor and also pre + post tumor exercise.

\section{Methods}

\section{Chemical}

Azoxymethane (AOM) was purchased from Sigma Aldrich Co. (St Louis, MO. USA). Ketamine and Xylazine were also purchased (Sigma Aldrich, USA). Total RNA was isolated with a miTotal RNA extraction kit (VIOGENE). Template cDNAs were synthesized using SuperScript III (Invitrogen). Quantitative RT-PCR was performed with a quantitative PCR mix (THUNDERBIRD; TOYOBO) using a realtime PCR detection system (CFX96; Bio-Rad Laboratories).

\section{Animals and study design}

Forty-eight Balb/c male mice (6-8 weeks old, Pasteur Institute of Iran) were randomly assigned to one of sex groups: Control $(C, n=8)$; healthy animals with neither tumor induction nor exercise training protocol, Exercise $(E, n=8)$; healthy animals received exercise protocol, Tumor induction $(T, n=8)$; animals received AOM for inducing colon cancer, Tumor + Exercise $(T E, n=8)$; animals with colon cancer underwent 6 weeks of the exercise training protocol [66] after tumor establishment, Exercise + Tumor (ET, $n=8)$; animals received exercise protocol one week before AOM consumption, and exercise + Tumor + exercise $(E T E, n=8)$; animals received exercise protocol one week before and after AOM consumption(about 15 weeks). Groups III - VI were weekly received AOM (as carcinogenic agent, $15 \mathrm{mg} / \mathrm{kg} \mathrm{s.c}$ ) in three consecutive weeks to induce colon cancer together with DSS 1\% for three consecutive weeks after the last injection of AOM [67]. All mice were housed individually and fed standard chow and water ad libitum and maintained on a 12:12 $\mathrm{h}$ light: dark cycle. The study was conducted in accordance with relevant Helsinki guideline and approved by the animal care and use committee of Iran University of Medical Sciences.

\section{Exercise training protocol}

All animals were equally randomized into the mentioned groups, and the body and colon weight, tumor size and colon length of animals in different groups were measured (Table 1). Prior to the initiation of the exercise training, the mice were assigned to the treadmill for 5 days. Acclimation entailed running at the end of their dark cycle $(0700)$ at gradually increasing speeds $(10,12,16$, and $18 \mathrm{~m} / \mathrm{min})$ and $0 \%$ inclination. Following the acclimation, the interval exercise training protocol was begun at $16-18 \mathrm{~m} / \mathrm{min}$, $0 \%$ grade, 10-14 min, 5 days/week for 6 weeks [68]. The exercise intensity (running speed) gradually was increased each week because of strong relationship between treadmill running speed and VO2 from sub maximal intensities up towards maximal levels in mouse and rat [69]. However, weekly increase in 
running speed during a training period could only be used as a guide to assess adaptation to the exercise training; therefore, control of the exercise intensity and the integrated effects of training require regulation of running speed based on serial measurements of V02max. All animals performed the same intensity and duration in each session. No electrical stimulation was used, and the mice were encouraged to run by a gentle tap on their tail or hindquarters. The untrained control animals were put on the switched-off treadmill during the same 6 weeks as the exercise-trained groups. The exercise training protocol was stopped $48 \mathrm{~h}$ before sacrifice [70].

\section{Depiction of tumors}

All animals were monitored closely for general health during the study period. Mice were weekly weighed and observed for evidence of rectal bleeding and death during study. At the end of the present study, almost $1.5 \mathrm{ml}$ of blood was intra-cardiacally aspirated under the general anesthesia. Blood samples were then centrifuged for $10 \mathrm{~min}$ at $4,000 \mathrm{rpm}$, and stored at $-80^{\circ} \mathrm{C}$ for further analysis. The animals were finally euthanized using cervical dislocation. A thorough necropsy was then made, and vital organs including the lung, the liver, the spleen, the small bowel intestinal, the brain and the colon were scrutinized for lesions and metastatic deposits. Tumors weight and their number, size and location were characterized.

\section{RNA extraction AND real-time quantitative PCR}

All tissue samples were immediately stored in liquid nitrogen and kept frozen at $-80{ }^{\circ} \mathrm{C}$ until RNA extraction. Isolation of RNA from all tumor samples and corresponding normal controls was simultaneously carried out using the miTotal RNA extraction kit (VIOGENE). The RNA concentration and purity of all samples were measured with NanoDrop (ND-1000 Spectrophotometer V3.5, USA), and cDNA synthesis was performed using Qiagen cDNA synthesis kit (Qiagen, Germany) according to the manufacturer's instructions. Briefly, $1 \mu \mathrm{g}$ of RNA was added to the reaction mixture (gDNA Wipeout Buffer, Quantiscript Reverse-Transcriptase, Quantiscript RT Buffer, RT Primer Mix, and RNase-free water) followed by incubation at $42^{\circ} \mathrm{C}$ for $15 \mathrm{~min}$. Reverse transcription was terminated at $95^{\circ} \mathrm{C}$ for 3 min and the RT products were then amplified by PCR on a GeneAmp PCR 9700 Thermocycler (Applied Biosystems) and then samples were stored at $-20^{\circ} \mathrm{C}$ until used for Real-time PCR analysis. Quantitative fluorescence PCR (QF-PCR) was used to amplify the MCT1, GLUT-1, PFK1, and P53 genes product in a 25 $\mu \mathrm{l}$ reaction system, with the use of SYBR-Green mix $(12.5 \mu \mathrm{l})$, upstream and downstream primers $(1 \mu \mathrm{l})$, cDNA $(2 \mu \mathrm{l})$ and RNase-free $\mathrm{H}_{2} \mathrm{O}(8.5 \mu \mathrm{l})$. Thermal cycling program was as follows: $94^{\circ} \mathrm{C}$ for $3 \mathrm{~min}$ followed by 30 cycles of $94{ }^{\circ} \mathrm{C}$ for $0.5 \mathrm{~min}, 54{ }^{\circ} \mathrm{C}$ for $1 \mathrm{~min}$, and $72{ }^{\circ} \mathrm{C}$ for $0.5 \mathrm{~min}$. GAPDH mRNA was used for normalization of the gene expression analysis. For primer design, the gene sequences were extracted from NCBI gene bank (www.NCBI.nIm.nih.gov). We used Primer 3 software to design PCR primers, and then submitted them to the selected BLAST databases for checkpoint. The primers were designed for amplification of 88 to 120 bp by the Rotor-Gene Q PCR device. The lyophilized primers were synthesized by Cinnagen Co. PCR primers sequences used for the amplification of the protein coding genes have been shown in Table 1. 
The $2^{-\triangle \Delta C T}$ method was used to evaluate the quantitative expression of P53 gene. All analyzes were performed separately for four sample groups [71].

Relative fold change in gene expression $=2^{-\Delta \Delta C T}$

$\Delta \mathrm{C}_{\mathrm{T}}=\mathrm{C}_{\mathrm{T} \text { target gene }}-\mathrm{C}_{\mathrm{T} \text { reference gene }}$

$\Delta \Delta \mathrm{C}_{\mathrm{T}}=\Delta \mathrm{C}_{\mathrm{T}}$ test sample $-\Delta \mathrm{C}_{\mathrm{T}}$ Control sample

\section{Measuring levels of MCT-1, GLUT-1 and PFK-1 protein}

For measuring the amount of protein MCT-1 (monocarboxylate transporter 1) in the colon tumor tissue from a commercial kit (ZellBio made in Germany with a sensitivity of $0.103 \mathrm{ng} / \mathrm{ml}$ ), the amount of GLUT-1 (glucose transporter 1) in the colon tumor tissue from commercial kit (ZellBio made in Germany with a sensitivity of $0.103 \mathrm{ng} / \mathrm{ml}$ ) and the protein content of the PFK1 (phosphorus kinase 1) enzyme in the colon tumor was also utilized from the commercial kit (ZellBio $\mathrm{GmbH}$, Ulm in Germany with a sensitivity of $0.095 \mathrm{ng} / \mathrm{ml}$ ) were used. All of this protein measurement in the tumor tissue was performed by ELISA methods.

\section{Statistical analysis}

Analysis of variance (ANOVA) and tukey test were used for comparison among groups. Values were represented as mean \pm SD. $P<0.05$ was considered to be statistically significant. Statistical analysis was done using SPSS statistical software, version 20.

\section{Abbreviations}

GLUT Glucose transporter

\section{Declarations}

\section{Authors' contributions}

All authors equally contribute in preparation of this manuscript

\section{Funding}

No funding.

\section{Availability of data and materials}

The datasets used and/or analyzed during the current study are available from the corresponding author on reasonable request. 


\section{Ethics approval and consent to participate}

The study protocol and informed consent form were consistent with the recommendations of the Declaration of Helsinki and approved by the ethics committee of Baqiyatallah University of Medical Science.

\section{Consent for publication}

This is not applicable.

\section{Competing interests}

There are no competing interests or conflicts of interest among any of the authors.

\section{References}

1. Soussi T, et al. High prevalence of cancer-associated TP53 variants in the gnomAD database: A word of caution concerning the use of variant filtering. Hum Mutat. 2019;40(5):516-24.

2. Jensen BW, et al. Change in weight status from childhood to early adulthood and late adulthood risk of colon cancer in men: a population-based cohort study. International Journal of Obesity. 2018;42(10):1797.

3. Bray F, et al., Global cancer statistics 2018: GLOBOCAN estimates of incidence and mortality worldwide for 36 cancers in 185 countries. CA: a cancer journal for clinicians, 2018. 68(6): p. 394424.

4. Cantor JR, Sabatini DM. Cancer cell metabolism: one hallmark, many faces. Cancer discovery. 2012;2(10):881-98.

5. Kroemer G, Pouyssegur J. Tumor cell metabolism: cancer's Achilles' heel. Cancer cell. 2008;13(6):472-82.

6. Martins SF, et al. Significance of glycolytic metabolism-related protein expression in colorectal cancer, lymph node and hepatic metastasis. BMC Cancer. 2016;16(1):535.

7. Bhattacharya B, Mohd MF, Omar, Soong R. The Warburg effect and drug resistance. Br J Pharmacol. 2016;173(6):970-9.

8. Jiang B. Aerobic glycolysis and high level of lactate in cancer metabolism and microenvironment. Genes Diseases. 2017;4(1):25-7.

9. Brophy S, et al. GLUT-1 expression and response to chemoradiotherapy in rectal cancer. International journal of cancer. 2009;125(12):2778-82.

10. Noguchi $Y$, et al. Suppression of facilitative glucose transporter 1 mRNA can suppress tumor growth. Cancer letters. 2000;154(2):175-82.

11. Chan DA, et al., Targeting GLUT1 and the Warburg effect in renal cell carcinoma by chemical synthetic lethality. Science translational medicine, 2011. 3(94): p. 94ra70-94ra70. 
12. Atsumi T, et al. High expression of inducible 6-phosphofructo-2-kinase/fructose-2, 6-bisphosphatase (iPFK-2; PFKFB3) in human cancers. Cancer research. 2002;62(20):5881-7.

13. Miranda-Gonçalves $V$, et al. Hypoxia-mediated upregulation of MCT1 expression supports the glycolytic phenotype of glioblastomas. Oncotarget. 2016;7(29):46335.

14. Andersen AP, et al. Roles of acid-extruding ion transporters in regulation of breast cancer cell growth in a 3-dimensional microenvironment. Mol Cancer. 2016;15(1):45.

15. Pinheiro $\mathrm{C}$, et al. Role of monocarboxylate transporters in human cancers: state of the art. J Bioenerg Biomembr. 2012;44(1):127-39.

16. Sonveaux $\mathrm{P}$, et al. Targeting lactate-fueled respiration selectively kills hypoxic tumor cells in mice. $J$ Clin Investig. 2008;118(12):3930-42.

17. Boidot R, et al. Regulation of monocarboxylate transporter MCT1 expression by p53 mediates inward and outward lactate fluxes in tumors. Cancer research. 2012;72(4):939-48.

18. Eriksson SE, et al. $p 53$ as a hub in cellular redox regulation and therapeutic target in cancer. $\mathrm{J} \mathrm{Mol}$ Cell Biol. 2019;11(4):330-41.

19. Basu S, et al. Mutant p53 controls tumor metabolism and metastasis by regulating PGC-1a. Genes Dev. 2018;32(3-4):230-43.

20. Devin JL, et al. Acute high intensity interval exercise reduces colon cancer cell growth. J Physiol. 2019;597(8):2177-84.

21. Weiner LS, et al., Breast cancer survivors reduce accelerometer-measured sedentary time in an exercise intervention. Journal of Cancer Survivorship, 2019: p. 1-9.

22. Weller S, Oliffe JL, Campbell KL. Factors associated with exercise preferences, barriers and facilitators of prostate cancer survivors. European journal of cancer care, 2019: p. e13135-e13135.

23. Liu N, et al. Effects of treadmill exercise on the expression of netrin-1 and its receptors in rat brain after cerebral ischemia. Neuroscience. 2011;194:349-58.

24. Moore SC, et al. Association of leisure-time physical activity with risk of 26 types of cancer in 1.44 million adults. JAMA internal medicine. 2016;176(6):816-25.

25. Dethlefsen $C$, et al. Exercise regulates breast cancer cell viability: systemic training adaptations versus acute exercise responses. Breast cancer research treatment. 2016;159(3):469-79.

26. Gatenby RA, Gillies RJ. Glycolysis in cancer: a potential target for therapy. Int J Biochem Cell Biol. 2007;39(7-8):1358-66.

27. Hojman P, et al. Molecular mechanisms linking exercise to cancer prevention and treatment. Cell Metabol. 2018;27(1):10-21.

28. Na HK, Oliynyk S. Effects of physical activity on cancer prevention. Ann N Y Acad Sci. 2011;1229(1):176-83.

29. De Oliveira EP, Burini RC. The impact of physical exercise on the gastrointestinal tract. Current Opinion in Clinical Nutrition Metabolic Care. 2009;12(5):533-8. 
30. Pedersen L, Christensen JF, Hojman P. Effects of exercise on tumor physiology and metabolism. The Cancer Journal. 2015;21(2):111-6.

31. Warburg O. On the origin of cancer cells. Science. 1956;123(3191):309-14.

32. Koelwyn GJ, et al. Exercise-dependent regulation of the tumour microenvironment. Nat Rev Cancer. 2017;17(10):620.

33. McTiernan A. Mechanisms linking physical activity with cancer. Nat Rev Cancer. 2008;8(3):205.

34. Jones LW, et al. Exercise and prognosis on the basis of clinicopathologic and molecular features in early-stage breast cancer: the LACE and pathways studies. Cancer research. 2016;76(18):5415-22.

35. Colbert LH, et al. Exercise effects on tumorigenesis in a p53-deficient mouse model of breast cancer. Med Sci sports Exerc. 2009;41(8):1597.

36. Aveseh M, Nikooie R, Aminaie M. Exercise-induced changes in tumour LDH-B and MCT1 expression are modulated by oestrogen-related receptor alpha in breast cancer-bearing BALB/c mice. J Physiol. 2015;593(12):2635-48.

37. Wallen $\mathrm{M}$, et al. Engage: preoperative exercise intervention for patients with prostate, colon and breast cancer. 2018.

38. Colditz GA, Cannuscio CC, Frazier AL. Physical activity and reduced risk of colon cancer: implications for prevention. Cancer Causes Control. 1997;8(4):649-67.

39. Végran $F$, et al. Lactate influx through the endothelial cell monocarboxylate transporter MCT1 supports an NF-KB/IL-8 pathway that drives tumor angiogenesis. Cancer research. 2011;71(7):255060.

40. De Saedeleer CJ, et al. Lactate activates HIF-1 in oxidative but not in Warburg-phenotype human tumor cells. PloS one. 2012;7(10):e46571.

41. Augoff K, Hryniewicz-Jankowska A, Tabola R. Lactate dehydrogenase 5: an old friend and a new hope in the war on cancer. Cancer letters. 2015;358(1):1-7.

42. Doherty JR, et al. Blocking lactate export by inhibiting the Myc target MCT1 Disables glycolysis and glutathione synthesis. Cancer research. 2014;74(3):908-20.

43. Draoui $\mathrm{N}$, et al. Antitumor activity of 7-aminocarboxycoumarin derivatives, a new class of potent inhibitors of lactate influx but not efflux. Mol Cancer Ther. 2014;13(6):1410-8.

44. Gohil K, Brooks GA. Exercise tames the wild side of the Myc network: a hypothesis. American Journal of Physiology-Endocrinology Metabolism. 2012;303(1):E18-30.

45. Hashimoto T, Hussien R, Brooks GA. Colocalization of MCT1, CD147, and LDH in mitochondrial inner membrane of $\mathrm{L} 6$ muscle cells: evidence of a mitochondrial lactate oxidation complex. American Journal of Physiology-Endocrinology Metabolism. 2006;290(6):E1237-44.

46. Kang R, Kroemer G, Tang D. The tumor suppressor protein p53 and the ferroptosis network. Free Radic Biol Med. 2019;133:162-8.

47. Ramani A. Effects of Exercise Training on the Blood Lactate Response to Acute Exercise in Cancer Survivors. 2019. 
48. Suzuki K, Matsubara H, Recent advances in p53 research and cancer treatment. BioMed Research International, 2011. 2011.

49. Kim S, An SSA. Role of p53 isoforms and aggregations in cancer. Medicine, 2016. 95(26).

50. Charrier-Savournin FB, et al., p21-Mediated nuclear retention of cyclin B1-Cdk1 in response to genotoxic stress. Molecular biology of the cell, 2004. 15(9): p. 3965-3976.

51. Watson JL, et al. Curcumin causes superoxide anion production and p53-independent apoptosis in human colon cancer cells. Cancer letters. 2010;297(1):1-8.

52. Lago CU, et al. p53, aerobic metabolism, and cancer. Antioxid Redox Signal. 2011;15(6):1739-48.

53. Kawauchi K, et al. p53 regulates glucose metabolism through an IKK-NF-KB pathway and inhibits cell transformation. Nat Cell Biol. 2008;10(5):611.

54. Santasusagna S, et al., miR-328 mediates a metabolic shift in colon cancer cells by targeting SLC2A1/GLUT1. Clinical and Translational Oncology, 2018. 20(9): p. 1161-1167.

55. Ambrosetti D, et al. The two glycolytic markers GLUT1 and MCT1 correlate with tumor grade and survival in clear-cell renal cell carcinoma. PloS one. 2018;13(2):e0193477.

56. Graziano F, et al. Glycolysis gene expression analysis and selective metabolic advantage in the clinical progression of colorectal cancer. The pharmacogenomics journal. 2017;17(3):258.

57. Elf SE, Chen J. Targeting glucose metabolism in patients with cancer. Cancer. 2014;120(6):774-80.

58. Cairns RA, Harris IS, Mak TW. Regulation of cancer cell metabolism. Nat Rev Cancer. 2011;11(2):85.

59. Ros S, et al., 6-Phosphofructo-2-kinase/fructose-2, 6-biphosphatase 4 is essential for p53-null cancer cells. Oncogene, 2017. 36(23): p. 3287.

60. Bensaad K, et al. TIGAR, a p53-inducible regulator of glycolysis and apoptosis. Cell. 2006;126(1):107-20.

61. Cheung EC, et al. TIGAR is required for efficient intestinal regeneration and tumorigenesis. Developmental cell. 2013;25(5):463-77.

62. Courneya KS, Friedenreich CM. Framework PEACE: an organizational model for examining physical exercise across the cancer experience. Ann Behav Med. 2001;23(4):263-72.

63. Courneya KS. Exercise in cancer survivors: an overview of research. Med Sci sports Exerc. 2003;35(11):1846-52.

64. Morgan T, et al., Effects of long-term exercise on human muscle mitochondria, in Muscle metabolism during exercise. 1971, Springer. p. 87-95.

65. SLATTERY ML, et al. Physical activity, diet, and risk of colon cancer in Utah. Am J Epidemiol. 1988;128(5):989-99.

66. Isanejad A, et al. MicroRNA-206, let-7a and microRNA-21 pathways involved in the anti-angiogenesis effects of the interval exercise training and hormone therapy in breast cancer. Life sciences. 2016;151:30-40.

67. Alizadeh AM, et al. Chemoprevention of azoxymethane-initiated colon cancer in rat by using a novel polymeric nanocarrier-curcumin. Eur J Pharmacol. 2012;689(1):226-32. 
68. Riggs CE Jr, et al. The effects of aerobic interval training on the left ventricular morphology and function of VLCAD-deficient mice. European journal of applied physiology. 2010;110(5):915-23.

69. Høydal MA, et al. Running speed and maximal oxygen uptake in rats and mice: practical implications for exercise training. European Journal of Cardiovascular Prevention Rehabilitation. 2007;14(6):75360.

70. Khori V, et al. Effects of exercise training together with tamoxifen in reducing mammary tumor burden in mice: Possible underlying pathway of miR-21. Eur J Pharmacol. 2015;765:179-87.

71. Dhup S, et al. Multiple biological activities of lactic acid in cancer: influences on tumor growth, angiogenesis and metastasis. Curr Pharm Design. 2012;18(10):1319-30.

\section{Tables}

Table 1

Real-time RT-PCR primer sequences

\begin{tabular}{|ll|}
\hline Genes & Primers \\
\hline MCT-1 & F: 5'-GGCCCGATTGGTCGCATGAGGGCT-3' \\
& R: 5'-GGCTGGGCAGTGGTAATTGGAGCT-3' \\
& F: 5'-CACATGCCTTGTTCTTTGCCAAG-3' \\
& R: 5'-TCTATACACAGCAGGGCAGGA-3' \\
PFK-1 & F: 5'-AGGAACGGCCAGATCGATA - 3' \\
& R: 5'-AGTCTCTGAGATCTTACCT-3' \\
& F: 5'-CACCTGCACAAGCGCCTCTCC-3' \\
& R: 5'-CTGCTGTCTCCAGACTCCTCTGTAGC-3' \\
GADPH & F: 5'-TCAACAGCAACTCCCACTCTTCC-3' \\
& R: 5'-ACCCTGTTGCTGTAGCCGTATTC-3' \\
\hline
\end{tabular}


Table 2

body and colon weight, tumor size and colon length of animals in different groups (Mean \pm SD).

\begin{tabular}{|c|c|c|c|c|}
\hline groups & $\begin{array}{l}\text { Body Weight } \\
\text { (g) }\end{array}$ & $\begin{array}{l}\text { Colon Weight } \\
\text { (mg/cm2) }\end{array}$ & $\begin{array}{l}\text { Colon length } \\
\text { (cm) }\end{array}$ & $\begin{array}{l}\text { Tumor Size } \\
(\mathrm{mm})\end{array}$ \\
\hline C & $17.69 \pm 1.23$ & $45.11 \pm 1.55$ & $15.07 \pm 1.33$ & - \\
\hline$E$ & $18.18 \pm 0.72$ & $45.85 \pm 0.76$ & $16.11 \pm 0.83^{b}$ & - \\
\hline $\mathrm{T}$ & $20.10 \pm 0.45^{a}$ & $51.21 \pm 2.08^{a}$ & $11.02 \pm 1.44^{\mathrm{a}}$ & $2.41 \pm 0.83$ \\
\hline TE & $19.02 \pm 1.51$ & $48.39 \pm 1.28$ & $13.45 \pm 1.94$ & $1.98 \pm 0.42^{b}$ \\
\hline ET & $19.57 \pm 1.73$ & $47.33 \pm 1.12$ & $14.66 \pm 0.76^{b}$ & $2.04 \pm 0.38$ \\
\hline ETE & $19.18 \pm 0.91$ & $46.95 \pm 2.13^{b}$ & $14.08 \pm 1.63^{b}$ & $1.89 \pm 0.53^{b}$ \\
\hline
\end{tabular}

Table 3

Effects of exercise training and tumor induction on the MCT-1, GLUT-1 and PFK-1 mRNA expression in mouse colon tumor cells.

\begin{tabular}{llll} 
Groups & $\begin{array}{l}\text { MCT-1 mRNA } \\
\text { (Change in fold to control) }\end{array}$ & $\begin{array}{l}\text { GLUT-1 mRNA } \\
\text { (Change in fold to control) }\end{array}$ & $\begin{array}{l}\text { PFK-1 mRNA } \\
\text { (Change in fold to control) }\end{array}$ \\
\hline C & $1.00 \pm 0.00$ & $1.00 \pm 0.00$ & $1.00 \pm 0.00$ \\
\hline E & $1.80 \pm 0.22^{\mathrm{b}}$ & $2.11 \pm 0.31^{\mathrm{b}}$ & $1.46 \pm 0.20^{\mathrm{b}}$ \\
\hline T & $8.20 \pm 1.32^{\mathrm{a}}$ & $7.38 \pm 1.52^{\mathrm{a}}$ & $9.11 \pm 1.12^{\mathrm{a}}$ \\
\hline TE & $4.31 \pm 0.81^{\mathrm{a}, \mathrm{b}}$ & $4.13 \pm 0.71^{\mathrm{a}, \mathrm{b}}$ & $5.80 \pm 0.89^{\mathrm{a}, \mathrm{b}}$ \\
\hline ET & $3.81 \pm 0.91^{\mathrm{a}, \mathrm{b}}$ & $5.51 \pm 0.82^{\mathrm{a}}$ & $5.23 \pm 0.66^{\mathrm{a}, \mathrm{b}}$ \\
\hline ETE & $2.44 \pm 0.75^{\mathrm{a}, \mathrm{b}}$ & $3.84 \pm 0.78^{\mathrm{a}, \mathrm{b}}$ & $3.43 \pm 0.84^{\mathrm{a}, \mathrm{b}}$
\end{tabular}

Data were show as means \pm SD. ${ }^{a}$ sign of significant difference compared with $\mathrm{C}$ group $(P<0.05)$, b sign of significant difference compared with T group $(P<0.05)$. Abbreviations: $\mathrm{C}$, control; $\mathrm{E}$, exercise training; T, tumor; TE, tumor + exercise training; ET, exercise training + tumor; and ETE, exercise training + tumor + exercise training.

\section{Figures}




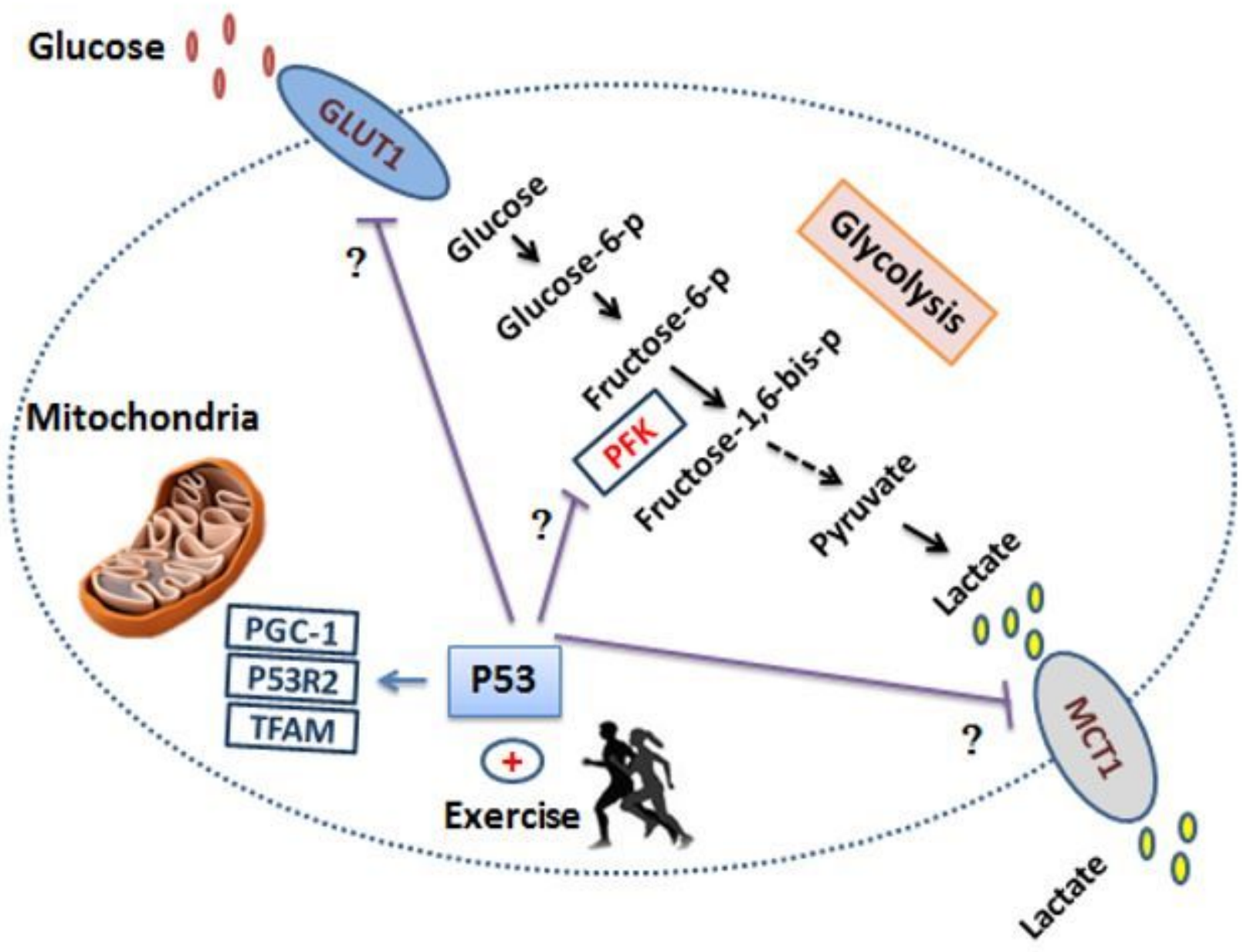

Figure 1

The regulation of cancer cell metabolism by p53. p53 regulates glycolysis and mitochondrial oxidative phosphorylation. p53 represses glycolysis through repressing the expression of GLUT1, PFK and MCT1. Physical activity and exercise are effective in upregulation of P53. 


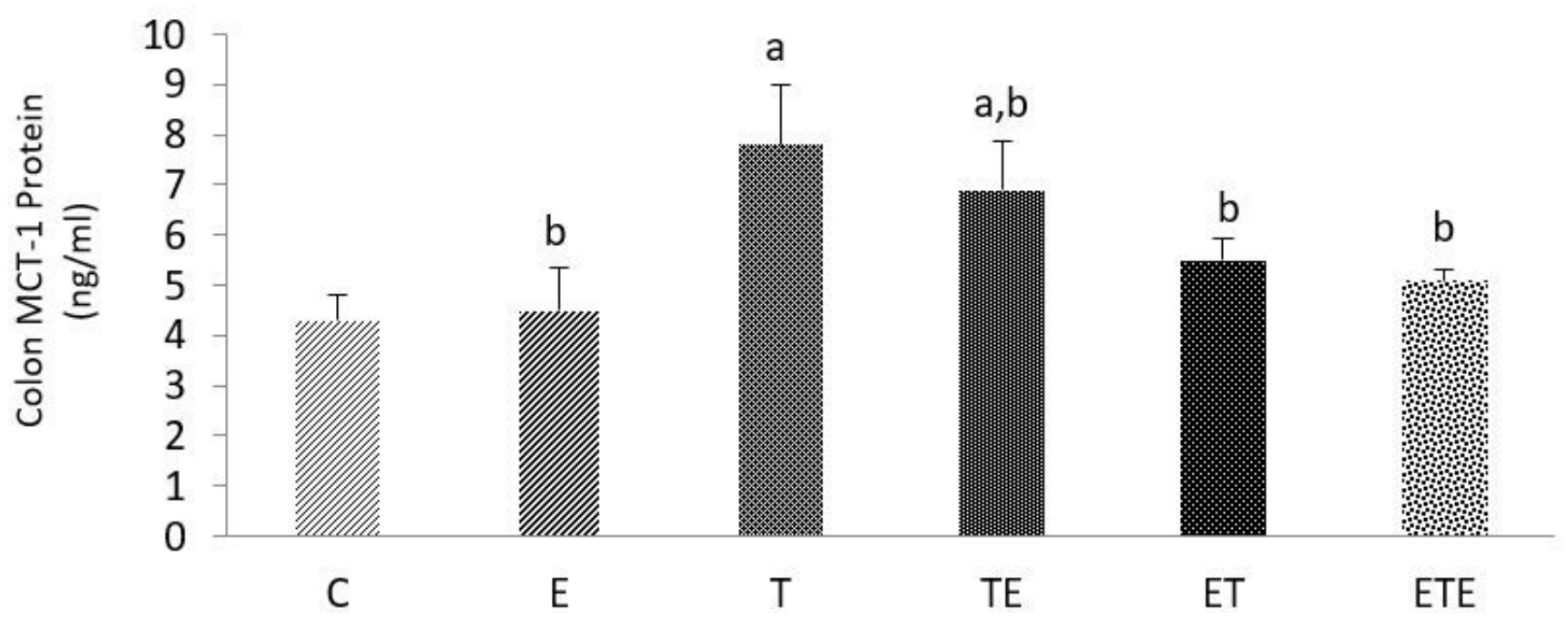

Figure 2

Effects of exercise training and tumor induction on the MCT-1 protein content in mouse colon. Data were show as means $\pm S D$. a sign of significant difference compared with $C$ group $(P<0.05)$, b sign of significant difference compared with $T$ group $(P<0.05)$. Abbreviations: $C$, control; $E$, exercise training; $T$, tumor; $\mathrm{TE}$, tumor + exercise training; $\mathrm{ET}$, exercise training + tumor; and ETE, exercise training + tumor + exercise training.

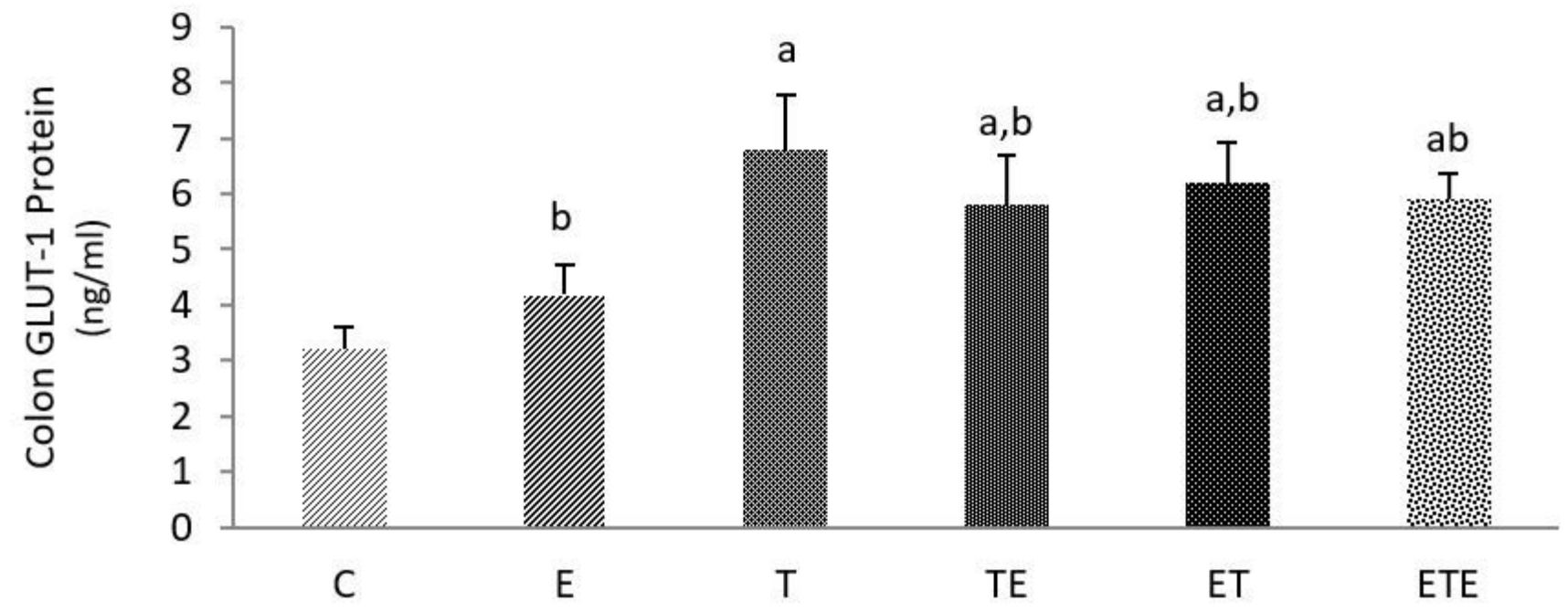

Figure 3 
Effects of exercise training and tumor induction on the GLUT-1 protein content in mouse. Data were show as means $\pm S D$. a sign of significant difference compared with $C$ group $(P<0.05)$, b sign of significant difference compared with $T$ group $(P<0.05)$. Abbreviations: $C$, control; $E$, exercise training; $T$, tumor; $T E$, tumor + exercise training; ET, exercise training + tumor; and ETE, exercise training + tumor + exercise training.

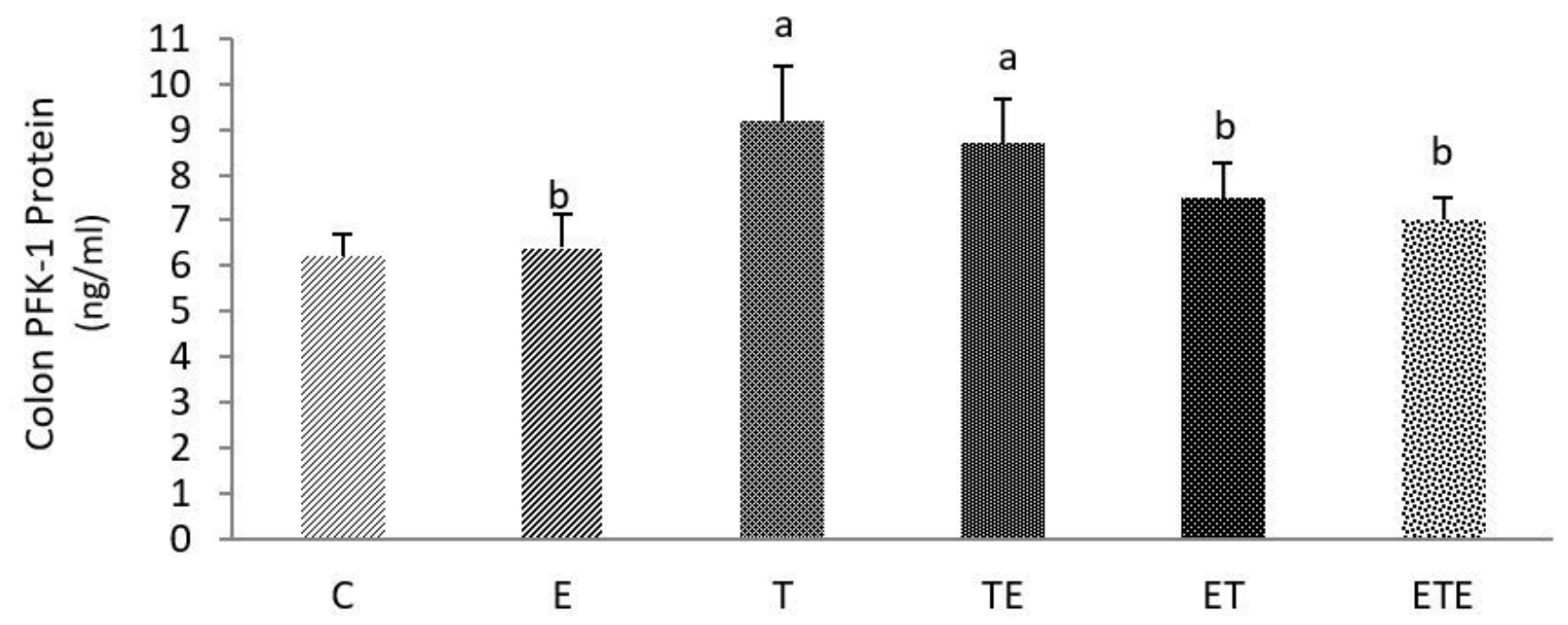

Figure 4

Effects of exercise training and tumor induction on the FPK-1 protein content in mouse colon. Data were show as means $\pm S D$. a sign of significant difference compared with $C$ group $(P<0.05)$, b sign of significant difference compared with $T$ group $(P<0.05)$. Abbreviations: $C$, control; $E$, exercise training; $T$, tumor; TE, tumor + exercise training; ET, exercise training + tumor; and ETE, exercise training + tumor + exercise training. 


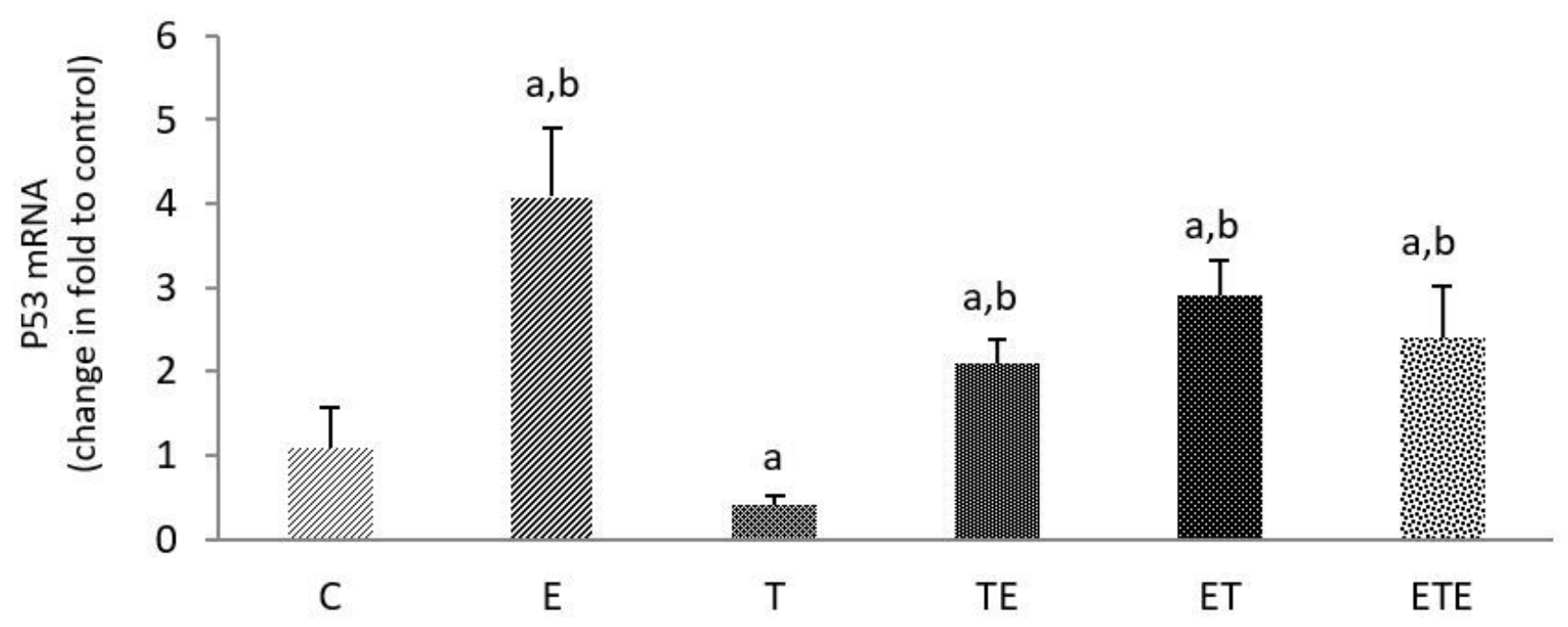

Figure 5

Effects of exercise training and tumor induction on the gene expression of P53 in mouse colon. Data were show as means $\pm S D$. a sign of significant difference compared with $C$ group $(P<0.05), b$ sign of significant difference compared with $T$ group $(P<0.05)$. Abbreviations: $C$, control; $E$, exercise training; $T$, tumor; $T E$, tumor + exercise training; $E T$, exercise training + tumor; and ETE, exercise training + tumor + exercise training. 